Neuroepidemiology 2009;32:30-31

DOI: $10.1159 / 000170089$

\section{The Impact of the DNA-Repair Gene MGMT in Malignant Gliomas}

Markus Hutterer

Neurooncology Group, Department of Neurology,

Medical University of Innsbruck, Innsbruck, Austria

Glioblastoma multiforme (GBM) is the most common and most aggressive primary brain tumor in adults. Despite advances in neurosurgical techniques, radiation and chemotherapy during the past three decades, little progress has been made in the treatment of malignant gliomas and therapy remains mostly palliative. In 2005, the EORTC/CAN-NCIC published the results of a large clinical trial examining the role of adjuvant temozolomide chemotherapy in the management of newly diagnosed GBM [1]. This study defined a new standard therapy for GBM, including maximal surgical tumor resection followed by temozolomide applied concomitantly and adjuvant to local field radiotherapy. This treatment results in a median overall survival time of only 9-15 months from the time of diagnosis [1].

One barrier to successful treatment of malignant glioma is resistance to alkylating agents such as chloroethylnitrosoureas (e.g. carmustine, lomustine, and fotemustine), procarbazine, and temozolomide. The cytotoxic activity of alkylating agents is largely mediated by the formation of O-methylguanine DNA adducts. Consequently, the primary mechanism of resistance is a function of the activity of the DNA repair enzyme O-methylguanine DNA methyltransferase (MGMT) in the tumor. The MGMT enzyme specifically removes the methyl group from the $\mathrm{O}^{6}$ position of guanine, thereby restoring the nucleotide to its native form without causing any DNA strand breaks. Furthermore, MGMT is a so-called suicide enzyme, because after the transfer of the alkyl group to an internal cysteine residue in the active site of $M G M T$, the enzyme is irreversibly inactivated, thus requiring de novo protein synthesis to maintain enzyme activity [2].

Epigenetic silencing of the MGMT gene by promoter methylation plays an important role in regulating MGMT expression [2]. In the last 10 years, several studies identified the MGMT promoter methylation status as a potential independent prognostic factor for survival in patients with GBM treated with alkylating agents and its association with a higher rate of clinical response [2]. In a companion tumor biology study to the EORTC/CAN-NCIC clinical trial, Hegi et al. [4] discovered the prognostic impact of the $M G M T$ promoter methylation status in patients with newly diagnosed GBM and treated with temozolomide. Furthermore, Martinez et al. [5] comparatively analyzed the MGMT promoter methylation status in 186 GBMs from patients with an expected me- dian survival and 9 from patients with long-term survival. The methylation rate in long-term GBM survivors was significantly higher, suggesting that MGMT promoter methylation is a hallmark of long-term survival GBMs and a specific characteristic of this intriguing GBM subtype [5].

In this issue of Neuroepidemiology, Zawlik et al. [6] studied the MGMT promoter methylation and polymorphisms in $371 \mathrm{GBMs}$ diagnosed at population level. MGMT methylation was observed in $44 \%$ GBMs, which is in line with data published by Hegi et al. [4]. Interestingly, MGMT methylation was observed with a higher frequency in secondary than in primary GBMs (73 vs. 43\%). Primary GBMs represent the majority of GBMs (95\%), develop rapidly de novo without clinical or histological evidence of a less malignant precursor lesion [7], mainly affect the elderly, and are genetically characterized by loss of heterozygosity 10q, EGFR amplification, $p 16^{I N K 4 a}$ deletion and PTEN mutations $[7,8]$. Secondary GBMs develop slowly through progression from low-grade diffuse astrocytoma (WHO grade II) or anaplastic astrocytoma (WHO grade III) and affect younger patients. In $60 \%$ of precursor low-grade astrocytomas, TP53 mutations are the most frequent and earliest detectable genetic alterations [9]. In this context, $\mathrm{Za}-$ wlik et al. [6] demonstrate that the frequency of TP53 G:C $\rightarrow$ A:T mutations in GBMs with MGMT methylation is significantly higher than that in GBMs without MGMT methylation. The authors conclude that MGMT methylation may be associated with susceptibility to acquire TP53 mutations. In addition, Zawlik et al. showed that the MGMT $143 \mathrm{Val}$ allele in GBMs is significantly less frequent than in a healthy European Caucasian population and is associated with longer survival compared to the MGMT 143 Ile allele. Therefore, not only the MGMT promoter methylaton status, but also MGMT polymorphisms may affect the prognosis of GBMs.

The findings reported by Zawlik et al. [6] are of interest to clinical neurooncology for several reasons. First, treatment strategies to overcome MGMT-mediated chemoresistance are being actively investigated. Since MGMT represents a suicide enzyme inactivated after each reaction, one strategy uses dose-intensified temozolomide schedules to deplete MGMT activity in tumor tissue [10]. Several studies have shown that prolonged exposure to temozolomide can deplete MGMT activity in blood cells. To date, however, it still remains unclear whether such dose-intensified regimes indeed effectively deplete MGMT activity in tumor tissue and whether they are more effective than the standard dosing regimen. Another strategy is the treatment with nontoxic pseudosubstrate inhibitors of MGMT (e.g. $\mathrm{O}^{6}$-benzylguanine). However, systemic application of MGMT inhibitors is limited by an increase in hematologic toxicity [10].

Secondly, in future the optimal treatment for GBM may vary substantially, depending on the MGMT expression status, which is in line with the conceptual development of more targeted personalized treatment strategies according to the molecular signatures of GBMs. Such developments are already reflected in the

\section{KARGER}

Fax +41613061234 E-Mail karger@karger.ch www.karger.com
(อ) 2008 S. Karger AG, Basel

0251-5350/09/0321-0030\$26.00/0 
design of recent clinical trials for GBMs, such as exploring the synergistic effects of the anti-angiogenic drug cilengitide compared to temozolomide monotherapy in patients with methylated GBMs only $[11,12$.

Given the central role of MGMT in alkylating agent resistance, MGMT is an ideal potential target for biochemical modulation of drug resistance. This is an area of intense ongoing research, which will hopefully result in further improvements in clinical outcomes.

\section{References}

1 Stupp R, Mason WP, van den Bent MJ, et al: Radiotherapy plus concomitant and adjuvant temozolomide for glioblastoma. N Engl J Med 2005;352:987-996.

-2 Esteller M, Garcia-Foncillas J, Andion E, et al: Inactivation of the DNArepair gene MGMT and the clinical response of gliomas to alkylating agents. N Engl J Med 2000;343:1350-1354.

- 3 Hegi ME, Liu L, Herman JG, et al: Correlation of $\mathrm{O}^{6}$-methylguanine methyltransferase (MGMT) promoter methylation with clinical outcomes in glioblastoma and clinical strategies to modulate MGMT activity. J Clin Oncol 2008;26:4189-4199.

-4 Hegi ME, Diserens AC, Gorlia T, et al: MGMT gene silencing and benefit from temozolomide in glioblastoma. N Engl J Med 2005;352:9971003.

5 Martinez R, Schackert G, Yaya-Tur R, et al: Frequent hypermethylation of the DNA repair gene MGMT in long-term survivors of glioblastoma multiforme. J Neurooncol 2007;83:91-93.
-6 Zawlik I, Vaccarella S, Kita D, Mittelbronn M, Franceschi S, Ohgaki H: Promoter methylation and polymorphisms of the MGMT gene in glioblastomas: a population-based study. Neuroepidemiology 2009;32:2129.

7 Ohgaki H, Dessen P, Jourde B, et al: Genetic pathways to glioblastoma: a population-based study. Cancer Res 2004;64:6892-6899.

$\checkmark 8$ Ohgaki H, Kleihues P: Genetic pathways to primary and secondary glioblastoma. Am J Pathol 2007;170:1445-1453.

-9 Ohgaki H, Kleihues P: Population-based studies on incidence, survival rates, and genetic alterations in astrocytic and oligodendroglial gliomas. J Neuropathol Exp Neurol 2005;64:479-489.

10 Wick W, Platten M, Weller M: New (alternative) temozolomide regimens for the treatment of glioma. Neuro Oncol 2008, E-pub ahead of print.

11 Nabors LB, Mikkelsen T, Rosenfeld SS, et al: Phase I and correlative biology study of cilengitide in patients with recurrent malignant glioma. J Clin Oncol 2007;25:1651-1657.

12 Reardon DA, Nabors LB, Stupp R, Mikkelsen T: Cilengitide: an integrin-targeting arginine-glycine-aspartic acid peptide with promising activity for glioblastoma multiforme. Expert Opin Investig Drugs 2008;17:1225-1235.

Markus Hutterer, MD

Neurooncology Group, Department of Neurology

Medical University of Innsbruck

Anichstrasse 35

AT-6020 Innsbruck (Austria)

Tel. +43 512504 82271, Fax +43512504 23852

E-Mail Markus.Hutterer@i-med.ac.at 